\title{
A NOTE ON BOUNDED-TRUTH-TABLE REDUCIBILITY
}

\author{
PATRICK C. FISCHER ${ }^{1}$
}

1. Introduction. In the 1944 paper of Post [1], the notions of oneone, many-one, bounded-truth-table, truth-table and Turing reducibility are introduced. For sets $A, B$ of positive integers let us abbreviate the statement " $A$ is one-one (many-one, bounded-truth-table) reducible to $B$ " by " $A \leqq_{1} B$ " ( $A \leqq_{m} B$," " $A \leqq_{{ }_{t t}} B$ ").

Bounded-truth-table and truth-table reducibilities are shown by Post to be distinct relations over the recursively enumerable, nonrecursive sets. In [2] and in [6], respectively, Dekker shows that one-one and many-one reducibilities differ on these sets and that truth-table and Turing reducibilities are distinct. This note will show that many-one and bounded-truth-table reducibilities also differ on these sets. Since the five reducibilities given are linearly ordered under implication (if $A \leqq_{1} B$, then $A \leqq_{m} B$, if $A \leqq_{m} B$, then $A \leqq_{b t t} B$, etc.), the conclusion that all five reducibilities are distinct on the recursively enumerable, nonrecursive sets will follow.

A second theorem will provide an example of a recursively enumerable bounded-truth-table degree of unsolvability which contains infinitely many distinct many-one degrees.

2. Preliminaries. Familiarity with $\S \S 1-8$ of [1] will be assumed and the notation therein will be used. Let $N$ denote the set of all positive integers. Let $A^{n}$ denote the Cartesian product of a set $A$ itself $n$ times. Thus, $A^{n}$ is the set of all ordered $n$-tuples $\left\langle x_{1}, x_{2}, \cdots, x_{n}\right\rangle$ of positive integers, all of whose components $\left\{x_{i}\right\}$ are in $A .^{2}$

REMARK. It is clear from the definition of bounded-truth-table reducibility $\left[1\right.$, p. 301] that for any set $A$ and any $n \in N, A^{n} \leqq_{b t t} A$ since $\left\langle x_{1}, x_{2}, \cdots, x_{n}\right\rangle \in A^{n} \equiv x_{1} \in A \& x_{2} \in A \& \cdots \& x_{n} \in A$. Also, $A \leqq_{1} A^{n}$; thus, the bounded-truth-table degree of unsolvability containing a set $A$ must contain $A^{n}$ for all $n \in N$.

Presented to the Society, October 28, 1963; received by the editors December 13, 1961 and, in revised form, September 6, 1962.

1 This paper was written while the author was a National Science Foundation Predoctoral Fellow. The author is indebted to the referee for his comments and suggestions.

${ }^{2}$ When considering certain recursively invariant properties of $A^{n}$, one usually works with indices of the $n$-tuples of $A^{n}$ under a fixed effective one-one mapping from $N^{n}$ into $N$. However, we shall let the notation $A^{n}$ stand for either the set of $n$-tuples of $A \times A \times \cdots \times A$ ( $n$ times) or the set of indices of those $n$-tuples, as context will make it clear which meaning is intended. 
3. Bounded-truth-table reducibility vs. many-one reducibility.

THEOREM 1. There exist two recursively enumerable, non-recursive sets $A$ and $B$ such that $A \leqq_{{ }_{b t}} B$ but not $A \leqq_{m} B$.

Proof. We choose a creative set $C$ and take $B$ to be the corresponding set $S_{1}$ of Post $\left[1\right.$, p. 304] and $A$ to be $B^{2}$. From the remark, $B^{2} \leqq{ }_{b t t} B$.

Post shows that $B\left(=S_{1}\right)$ has the following properties:

(i) $B$ is simple (in particular, $B$ is not creative).

(ii) For any $n \in N, n \in C \equiv 2^{n}+1 \in B \& 2^{n}+2 \in B \& \cdots \& 2^{n+1} \in B$. Now suppose $B^{2} \leqq_{m} B$, i.e., suppose there is a recursive function $g(x, y)$ of two variables such that $\langle x, y\rangle \in B^{2} \equiv x \in B \& y \in B$ $\equiv g(x, y) \in B$. Then we can reduce $C$ many-one to $B$ as follows: for any $n \in N$,

$$
\begin{aligned}
n \in C \equiv & 2^{n}+1 \in B \& 2^{n}+2 \in B \& 2^{n}+3 \in B \& \cdots \& 2^{n+1} \in B \\
\equiv & g\left(2^{n}+1,2^{n}+2\right) \in B \& 2^{n}+3 \in B \& \cdots \& 2^{n+1} \in B \\
\equiv & g\left(g\left(2^{n}+1,2^{n}+2\right), 2^{n}+3\right) \in B \& \cdots \& 2^{n+1} \in B \\
& \cdot \cdot \cdot \cdot \cdot \cdot \cdot \cdot \cdot \cdot \cdot \cdot \cdot \cdot \cdot \cdot \cdot \cdot \cdot \cdot \\
\equiv & g\left(g\left(\cdots g\left(g\left(2^{n}+1,2^{n}+2\right) 2^{n}+3\right), \cdots, 2^{n+1}-1\right), 2^{n+1}\right) \in B .
\end{aligned}
$$

We define $f(n)$ to be the recursive function which, given $n$, applies $g(x, y) 2^{n}-1$ times as indicated above. Then $n \in C \equiv f(n) \in B$. By a well-known result $[2$, p. $500 ; 3$, p. 100$] B$ must then be creative, contradicting the fact that $B$ is simple. Therefore $B^{2}{ }_{m} B$.

Theorem 2. There exists a simple set $B$ such that the sets $B, B^{2}$, $B^{3}, \ldots$ lie in different many-one degrees of unsolvability.

Proof. Take $B$ as in Theorem 1. Choose positive integers $m$ and $k$ and consider $B^{m}$ and $B^{m+k}$. Clearly, $B^{m} \leqq_{m} B^{m+1} \leqq_{m} B^{m+k}$. Suppose $B^{m+k}$ and $B^{m}$ are in the same many-one degree. Then $B^{m+1}$ is also in that degree and, in particular, $B^{m+1} \leqq_{m} B^{m}$. Therefore, there exists a recursive mapping $h$ from $N^{m+1}$ into $N^{m}$ such that if $h$ takes $\left\langle x_{1}, x_{2}, \cdots, x_{m+1}\right\rangle$ into $\left\langle y_{1}, y_{2}, \cdots, y_{m}\right\rangle$, then $\left\langle x_{1}, x_{2}, \cdots, x_{m+1}\right\rangle$ $\in B^{m+1} \equiv\left\langle y_{1}, y_{2}, \cdots, y_{m}\right\rangle \in B^{m}$, i.e., $x_{1} \in B \& x_{2} \in B \& \cdots \& x_{m+1} \in B$ $\equiv y_{1} \in B \& y_{2} \in B \& \cdots \& y_{m} \in B$. Again, we know that $n \in C \equiv 2^{n}+1$ $\in B \& 2^{n}+2 \in B \& \cdots \& 2^{n+1} \in B$, and by applying $h 2^{n}-m$ times we have an effective process giving outputs $z_{1}(n), z_{2}(n), \cdots, z_{m}(n)$ such that $n \in C \equiv z_{1}(n) \in B \& z_{2}(n) \in B \& \cdots \& z_{m}(n) \in B$ $\equiv\left\langle z_{1}(n), \quad z_{2}(n), \cdots, \quad z_{m}(n)\right\rangle \in B^{m}$. (If $2^{n}<m$, then $n \in C$ $\equiv\left\langle 2^{n}+1,2^{n}+2, \cdots, 2^{n+1}, b, b, \cdots, b\right\rangle \in B^{m}$ where $b$ is some fixed 
member of $B$ occurring $m-2^{n}$ times in the $m$-tuple.) Therefore, $B^{m}$ is creative. This, however, is impossible, for $B^{m} \leqq_{b t t} B$, and by a wellknown result of Post $[1$, p. 304] no creative set is bounded-truth-table reducible to a simple set.

CoROLlaRy. There exists at least one bounded-truth-table degree of unsolvability for recursively enumerable sets which is partitioned into $\boldsymbol{\aleph}_{0}$ many-one degrees of unsolvability.

Proof. The corollary follows directly from Theorem 2 and from the Remark.

\section{REFERENCES}

1. E. L. Post, Recursively enumerable sets of positive integers and their decision problems, Bull. Amer. Math. Soc. 50 (1944), 284-316.

2. J. Dekker, Two notes on recursively enumerable sets, Proc. Amer. Math. Soc. 4 (1953), 495-501.

3. J. Myhill, Creative sets, Z. Math. Logik Grundlagen Math. 1 (1955), 97-108.

4. H. Rogers, Jr., The present theory of Turing machine computability, J. Soc. Indust. Appl. Math. 7 (1959), 114-130.

5. J. Shoenfield, Quasicreative sets, Proc. Amer. Math. Soc. 8 (1957), 964-967.

6. J. Dekker, $A$ theorem on hypersimple sets, Proc. Amer. Math. Soc. 5 (1954) 791-796.

MassachusetTs Institute of Technology 